\title{
Exploring the Universe with Ultra High Energy Cosmic Rays
}

\author{
Lorenzo Perrone $^{*, a}$ for the Pierre Auger Collaboration ${ }^{b}$ \\ ${ }^{a}$ Università del Salento and INFN Lecce, Italy \\ ${ }^{b}$ Observatorio Pierre Auger, Av. San Martín Norte 304, 5613 Malargüe, Argentina \\ E-mail: lorenzo.perrone@le.infn.it \\ Full author list: http://www.auger.org/archive/authors_2015_08.html
}

\begin{abstract}
Ultra High Energy Cosmic Rays (UHECR) are messengers of the most powerful astrophysical sources in the universe and they offer the unique chance of investigating particle interactions over an energy range well beyond the one covered by present ground-based particle accelerators. Despite several progresses have been made in the last decade, the understanding of the nature of UHECR and of their origin remains an open science case. A review of selected results from the largest UHECR experiments, namely The Pierre Auger Observatory and the Telescope Array, is presented with the emphasis given to the measurements of energy spectrum, arrival directions, chemical composition, and the search for photons and neutrinos as primary particles. Connection to hadronic physics and open issues will also be discussed along with the perspectives for near future measurements.
\end{abstract}

XXVII International Symposium on Lepton Photon Interactions at High Energies

17-22 August 2015

Ljubljana, Slovenia

${ }^{*}$ Speaker. 


\section{Introduction}

The Pierre Auger Observatory [1] is located in the Province of Mendoza, Argentina, and consists of a surface array (SD) of about $3000 \mathrm{~km}^{2}$ overlooked by 27 air fluorescence telescopes (FD) grouped in four sites, which together provide a powerful instrument for air shower reconstruction. The SD comprises 1600 water-Cherenkov detectors separated by $1500 \mathrm{~m}$ in a triangular grid, plus a smaller nested array of 49 additional detectors spaced by $750 \mathrm{~m}$ covering an area of $24 \mathrm{~km}^{2}$.

The Telescope Array [2] is located in the high desert in Millard County, Utah, USA over a region of $500 \mathrm{~km}^{2}$. It consists of more than 500 scintillator detectors of $3 \mathrm{~m}^{2}$ each, located on a $1.2 \mathrm{~km}$ square grid. In addition, there are three telescope stations on a $30 \mathrm{~km}$ triangle. They are instrumented with 12-14 telescopes each. Both experiments use a hybrid detection technique combining information from a surface array, measuring the lateral distributions of secondary particles at the ground, and fluorescence telescopes, observing the shower longitudinal profile. This design enhances the reconstruction capability and the measurement accuracy with respect to the individual detector components. This review is based on the results presented at the last International Cosmic Ray Conference [3, 4].

\section{The energy spectrum}

The energy spectrum is a key observable for the understanding of the origin and nature of ultra-high energy cosmic rays (UHECRs). The accurate measurement of the spectrum presents a challenge for astrophysical modelling of origin and propagation of UHECRs. Two relevant spectral features have been established beyond doubt by the Auger and TA experiments: the hardening in the spectrum at about $5 \times 10^{18} \mathrm{eV}$ (the ankle), and a strong suppression of the flux at the highest energies. The fluorescence detection technique can provide a nearly calorimetric measurement of the primary energy, almost independent of the assumptions on hadronic interaction models at the highest energies. This property is exploited to calibrate the surface detector estimators of primary energy, thus combining the quality of the energy measurement provided by the fluorescence detector with the benefit of a duty cycle of almost $100 \%$ as provided by the surface detector.

The latest results of the energy-spectrum measurements by the Pierre Auger Collaboration are discussed in details in [5]. A high-quality subset of hybrid events detected simultaneously by the FD and at least one station of the SD is used to calibrate the SD energy estimators with the energies measured with the FD. To ensure good energy reconstruction, only events that satisfy strict quality criteria are accepted [7]. The shower energy estimated with the FD has a total systematic uncertainty of $14 \%$ [6]. The calibrations are shown in Fig. 1, left panel. $S_{35}$ and $S_{38}$ are the energy estimators for the so-called vertical showers (with zenith angle $\theta<60^{\circ}$ ) recorded by SD-750 $\mathrm{m}$ (the infill array) and SD-1500 m, respectively: they are the signals at $450 \mathrm{~m}$ and $1000 \mathrm{~m}$ from the shower core corrected for atmospheric absorption. $\mathrm{N}_{19}$ is the energy estimator for the so-called horizontal events $\left(60^{\circ}<\theta<80^{\circ}\right)$ : it is related to the muon content and thus directly connected to the primary energy. The three correlations are well described by simple power-law functions $\mathrm{E}_{F D}=\mathrm{A} \mathrm{X}^{B}$ with $\mathrm{X}=\mathrm{S}_{38}, \mathrm{~S}_{35}, \mathrm{~N}_{19}$. The four independent measurements of the energy spectrum, based on an exposure now larger than $50000 \mathrm{~km}^{2}$, are shown in Fig. 1, right. Data from the SD-750 $\mathrm{m}$ allow for the determination of the energy spectrum down to $10^{17} \mathrm{eV}$. The SD-1500 m vertical 


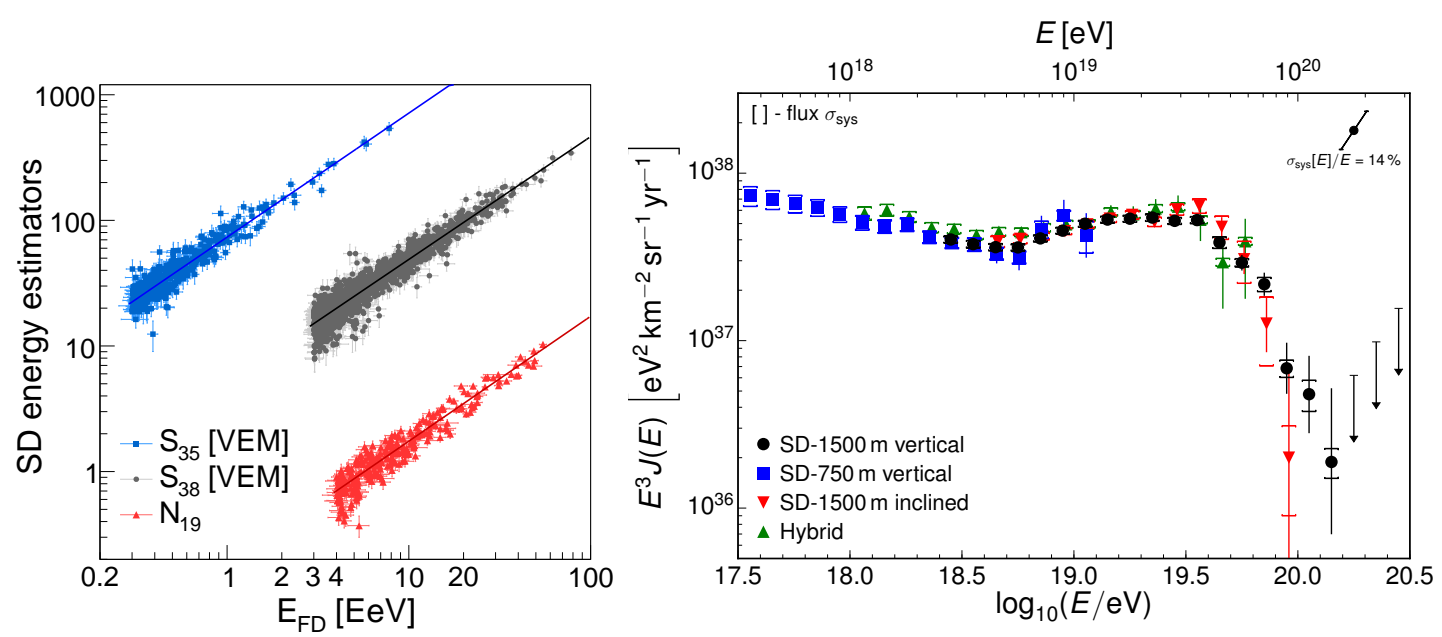

Figure 1: Left: Correlation between the energy estimators (see text) and the energy FD energy. $S_{38}$ and $S_{35}$ are given in units of Vertical Equivalent Muon or VEM, corresponding to the signal produced by a vertical muon traversing the detector through its center. Since $N_{19}$ is a scaling factor it is dimensionless. Right: energy spectra derived from SD and hybrid data recorded at the Pierre Auger Observatory. The error bars represent statistical uncertainties. See [5] for details.

data are crucial above the energy of full trigger efficiency of $3 \times 10^{18} \mathrm{eV}$ up to the highest energies, with horizontal events contributing above $4 \times 10^{18} \mathrm{eV}$ and providing an independent measurement in this energy range. Hybrid data bridge those from the two SDs, between $10^{18} \mathrm{eV}$ and $10^{19.6} \mathrm{eV}$. The four independent measurements of the energy spectrum of cosmic rays are then combined using a method that takes into account the systematic uncertainties of the individual measurements. The Auger combined spectrum is shown in Fig 2, left [5]. The features of the spectral shape around the ankle are characterized using a fit to a broken power-law function with different spectral indices. A smooth exponential suppression is included to describe the behaviour at the highest energies. The ankle is found to be at $E_{\text {ankle }}=(4.8 \pm 0.1 \pm 0.8) \times 10^{18} \mathrm{eV}$. The spectral slope below the ankle is $\gamma_{1}=3.29 \pm 0.02 \pm 0.05$, while above the ankle it is $\gamma_{2}=2.60 \pm 0.02 \pm 0.10$. The energy at which the differential flux falls to one-half of the value of the power-law extrapolation is $E_{1 / 2}=$ $(42.1 \pm 1.7 \pm 7.6) \times 10^{18} \mathrm{eV}$.

The existence of the low energy ankle is confirmed also by the TA collaboration with the low energy extension fluorescence detectors [8]. The energy spectrum obtained by combining all measurements available at TA is based on a total exposure of about $6.300 \mathrm{~km}^{2} \mathrm{sr}$ yr. The TA and Auger combined spectra are compared in Fig. 2 (right), taken from [9]. The ankle at $\sim 5 \times 10^{18} \mathrm{eV}$ and the break at $\sim 6 \times 10^{19} \mathrm{eV}$ are also evident in TA data. The two measurements exhibit a good agreement at energies below $2 \times 10^{19} \mathrm{eV}$ with a difference in the flux of about $20 \%$. A sizeable discrepancy, only marginally consistent with systematic uncertainties, is instead found in the region of the cut-off. As shown in the figure, the position of $E_{1 / 2}$, is different for the two experiments, leading to a different description of the suppression occurring at the end of the UHECRs spectrum. The systematic uncertainties quoted by Auger include the contribution from the uncertainty in the energy scale. For TA only the statistical error is reported. A common effort by the two collaborations is ongoing to study the origin of this difference [10]. As Auger and TA 

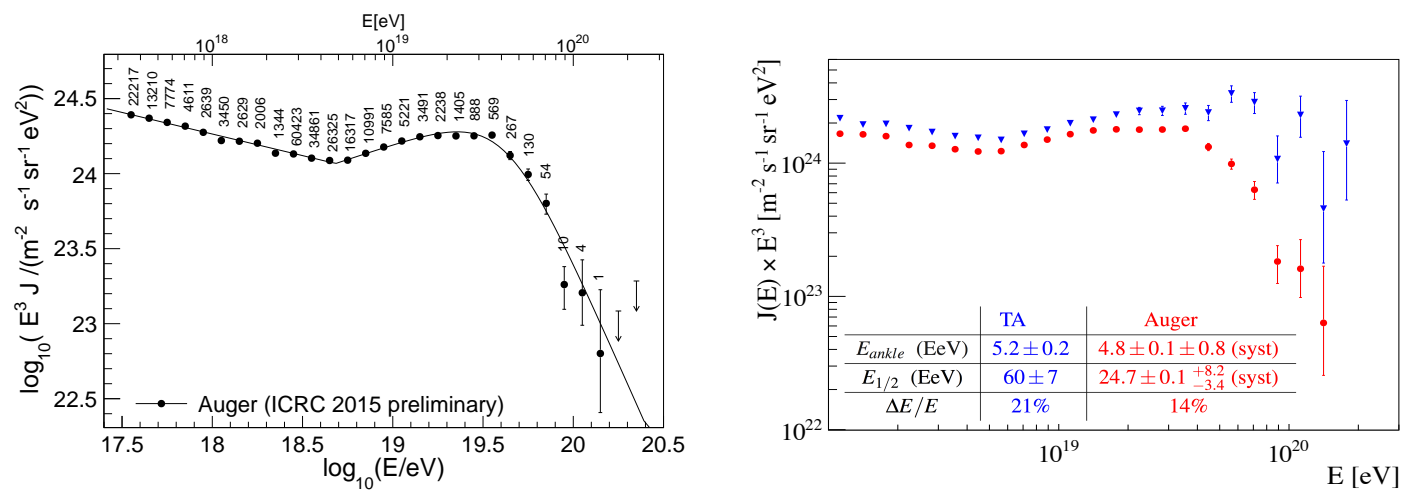

Figure 2: Left: the combined energy spectrum of cosmic rays as measured by the Auger Observatory, fitted with a flux model (see text). Only statistical uncertainties are shown. The systematic uncertainty on the energy scale is $14 \%$. The number of events is given above the points. The upper limits correspond to the 84\% C.L.. Right: Comparison between Auger [5] and TA [8] spectra, taken from [9].

observe different parts of the sky for most of the time, a search for a declination dependence of the flux of cosmic rays was performed. No significant variation in the flux measured with the Auger SD in four declination bands were found that could account for the discrepancy between spectra measured from different hemispheres[5]. The differences found between the measurements in two separate declination bands are compatible with the variations expected from a dipolar modulation of the flux.

\section{Mass composition}

The nuclear composition of ultra-high energy cosmic rays is one of the key observables to understand their origin. One of the most robust and precise observables to infer the composition from air-shower measurements is the atmospheric depth at which the shower reaches its maximum size, $X_{\max }$. Currently, the Pierre Auger Observatory and the Telescope Array measure $X_{\max }$ using fluorescence detectors. Despite the use of the same detection principle, a direct comparison of the data published by both collaborations is not straightforward.

For the first time the Pierre Auger Collaboration presented $\left\langle X_{\max }\right\rangle$ and $\sigma\left(X_{\max }\right)$ measurements covering nearly three decades of energy [11] using data obtained with the High Elevation Auger Telescopes (HEAT) enhancement. The HEAT telescopes cover a field of view ranging from $30^{\circ}$ to $60^{\circ}$ of elevation and are located next to one of the standard fluorescence detector sites (Coihueco). The combination of HEAT and Coihueco telescopes allows us to extend the energy range down to $10^{17} \mathrm{eV}$. The knowledge of the composition of cosmic rays in the energy range of 0.1 to $1 \mathrm{EeV}$ is a key ingredient to identify a possible transition from galactic to extra-galactic sources and for understanding the nature of the energy-spectrum features. More than 18000 events collected by the standard FD telescopes above $10^{17.8} \mathrm{eV}$ have been supplemented by about 5500 events collected with HEAT in coincidence with Coihueco (HeCo). A shower is reconstructed accurately only if its $X_{\max }$ is within the telescope field of view. Shallow or deep events are more likely to have their $X_{\max }$ values outside it and have larger chances to be excluded from the analysis. A fiducial cut aimed at 

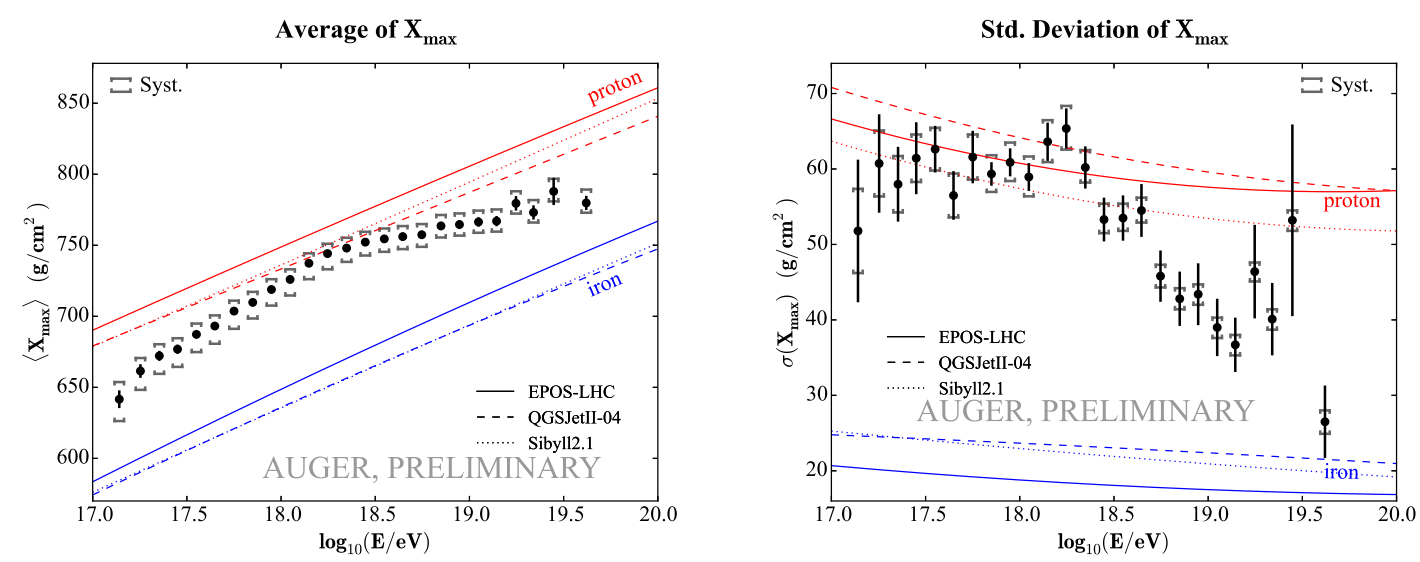

Figure 3: The mean (left) and the standard deviation (right) of the measured $\mathrm{X}_{\max }$ distributions (combining $\mathrm{HeCo}$ and standard data sets) as a function of energy compared to air-shower simulations for proton and iron primaries.

the rejection of events with biased reconstruction is then applied following the strategy described in detail in [12].

Between $10^{17.0}$ and $10^{18.3} \mathrm{eV}\left\langle X_{\max }\right\rangle$ increases by around $85 \mathrm{~g} \mathrm{~cm}^{-2}$ per decade of energy (see Fig. 3, left). This value is larger than the one expected for a constant mass composition ( $\sim 60 \mathrm{~g} \mathrm{~cm}^{-2} /$ decade) and it indicates that the mean primary mass is getting lighter. Around $\approx$ $10^{18.3} \mathrm{eV}$ the observed rate of change of $\left\langle X_{\max }\right\rangle$ becomes significantly smaller $\left(\sim 26 \mathrm{~g} \mathrm{~cm}^{-2} /\right.$ decade $)$ indicating that the composition is becoming heavier. The standard deviation starts to decrease at around the same energy $\approx 10^{18.3} \mathrm{eV}$ (see Fig. 3, right). The first two moments of the $X_{\max }$ distribution $\left(\left\langle X_{\max }\right\rangle\right.$ and $\left.\sigma\left(X_{\max }\right)\right)$ are related to the first two moments of the distribution of the logarithm of masses of primary particles $\left(\langle\ln A\rangle\right.$ and $\left.\sigma^{2}(\ln A)\right)$ as shown in [14]. Using EPOS-LHC and QGSJetII-04 as hadronic interaction models similar trends with energy for $\langle\ln A\rangle$ and $\sigma^{2}(\ln A)$ are observed in Auger hybrid data [11]. The primary mass is decreasing reaching the minimal values at around $10^{18.3} \mathrm{eV}$ and starts to increase for the higher energies. The spread of the masses is almost constant till $\approx 10^{18.3} \mathrm{eV}$ and then it starts to decrease, together with $\langle\ln A\rangle$. That might be an indication that the relative fraction of protons becomes smaller for the energies above $\approx 10^{18.3} \mathrm{eV}$ (see[13]). In particular, the study of $\sigma^{2}(\ln A)$ indicates that Auger data disfavor the hadronic model QGSJetII-04[12,11]. The implication of the distributions of $X_{\max }$ have been studied in detail by the Pierre Auger Collaboration by considering different assumptions on composition and on hadronic interaction models [13]. Regardless of what interaction model is assumed, Auger data are not well described by a mix of protons and iron nuclei over most of the energy range. Acceptable fits can be obtained when intermediate masses are included.

The TA Collaboration published values of the average shower maximum, $\left\langle X_{\max }\right\rangle$, obtained from $X_{\max }$ distributions that include detector effects such as the selection efficiency and acceptance [16]. The interpretation of the data is made possible by the comparison of the Monte-Carlo prediction for proton and iron nuclei folded with the same detector resolution and efficiency. The corresponding values of $\left\langle X_{\max }\right\rangle$ are presented in Fig. 4 left, together with predictions from airshower simulations for proton- and iron-initiated showers. SIBYLL2.1, the only hadronic interac- 

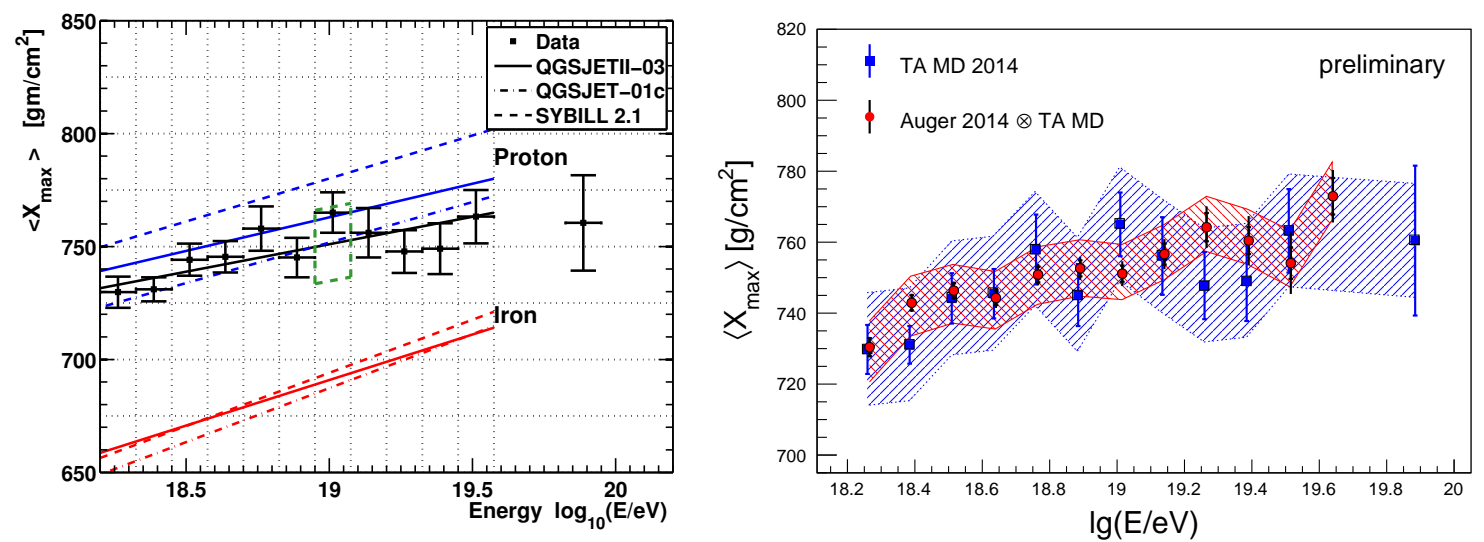

Figure 4: Left: $\left\langle X_{\max }\right\rangle$ as measured by Telescope Array Collaboration [16]. The coloured lines are for predictions of air-shower simulations. The black line on the right panel is a straight-line fit to the TA data. The systematic uncertainty is indicated by the green dashed box. Right: Comparison of $\left\langle X_{\max }\right\rangle$ as measured with the MD of TA (blue squares) and the $\left\langle X_{\max }\right\rangle$ of the Auger data folded with the MD acceptance (red circles). The data points are slightly shifted horizontally for better visibility. The coloured bands show the systematic uncertainties of the $X_{\max }$ scales of each experiment. See [15] for details.

tion model used by both collaborations, provides a common reference in these plots. This analysis is the result of a common effort of the Pierre Auger and TA Collaborations with the aim of providing a direct comparison of the $\left\langle X_{\max }\right\rangle$ measurements taking into account the different approaches of each collaboration [15]. Indirect comparisons of TA and Auger results using a conversion of $\left\langle X_{\max }\right\rangle$ to the average logarithmic mass were published earlier in [17]. The disadvantage of indirect comparisons is that they depend on the particular hadronic interaction model that is used. The current analysis was performed by taking into account that the $\left\langle X_{\max }\right\rangle$ published by Auger are corrected for detector effects, whereas those published by TA include detector effects. The resulting effect of the folding of the parametric Auger distributions with the TA detector response is that the mean value after the application of the TA detector response is smaller than the generated mean. The $\left\langle X_{\max }\right\rangle$ as measured by TA using the Middle Drum (MD) fluorescence telescope and the Auger result folded with the TA acceptance are shown in Fig. 4 right. Their compatibility is quantified with a bin-by-bin comparison excluding the highest-energy data points of each experiment which are at different energies. Using only the statistical uncertainties yields a $\chi^{2} /$ Ndf of $10.7 / 11$ with $P\left(\chi^{2} \geq 10.7 \mid 11\right)=0.47$. The average difference of the data points is $(2.9 \pm 2.7$ (stat.) \pm 18 (syst.) $) \mathrm{g} / \mathrm{cm}^{2}$ with a $\chi^{2} / \mathrm{Ndf}$ of $9.5 / 10(P=0.48)$.

It can be concluded that the two data sets are in excellent agreement, even without accounting for the respective systematic uncertainties on the $X_{\max }$ scale. However, this study did not take into account a possible difference in the energy scale of the two experiments. The comparison of the energy spectra at the ankle region suggests that the energy scale of TA is about $13 \%$ higher than the one of the Pierre Auger Observatory [10]. Nevertheless, it is to be expected that the increased difference between the two data sets once the energy scale shift is taken into account will be much smaller than the systematic uncertainties on the $X_{\max }$ scale of $\leq 10 \mathrm{~g} / \mathrm{cm}^{2}$ and $16 \mathrm{~g} / \mathrm{cm}^{2}$ for the Auger and TA analyses respectively. 

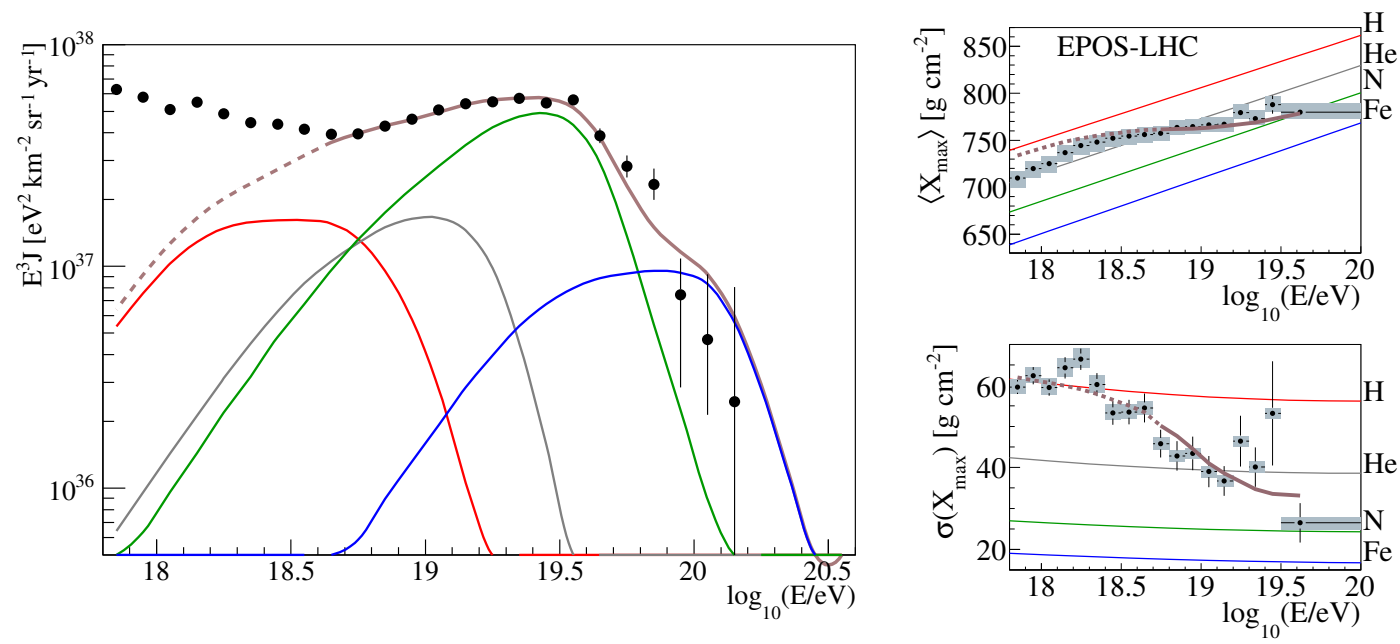

Figure 5: Left: Simulated energy spectrum of UHECRs at the top of the Earth's atmosphere according to the best-fit scenario (brown) along with Auger data points [20]. Individual nuclei spectra are also shown: pure ${ }^{1} \mathrm{H}$ (red), ${ }^{4} \mathrm{He}$ (grey), ${ }^{14} \mathrm{~N}$ (green) and ${ }^{56} \mathrm{Fe}$ (blue). Right: average (top) and standard deviation (bottom) of the $X_{\max }$ distribution as predicted in this scenario assuming EPOS-LHC. Only the energy range where the brown lines are solid is included in the fit. See [18] for details.

\section{Astrophysical interpretation}

A combined fit of both flux and composition of ultra-high energy cosmic rays as measured by the Pierre Auger Observatory has been performed for energies above $5 \times 10^{18} \mathrm{eV}$ [18]. A simple astrophysical model consisting of identical sources uniformly distributed in a comoving volume has been adopted. Proton, helium, nitrogen and iron nuclei are injected at the source assuming a power law spectrum and a rigidity dependent exponential cut-off.

Cosmic rays are propagated from the sources to the observer using the SimProp code [19]. The free parameters of the fit are the normalization of the flux at the source, its spectral index $\gamma$ and its cutoff rigidity $R_{\text {cut }}$, as well as the elemental fractions at the source. The simulated spectra and the mean/variance of the simulated $X_{\max }$ distributions corresponding to the best fit are shown in Fig.5, left and right top/bottom, respectively, together with data. The spectrum is best fitted by a succession of cutoffs of the different group of elements, with $R_{\text {cut }}=10^{18.67 \pm 0.03} \mathrm{~V}$, thus indicating that the flux at Earth could be limited by the maximum energy at the source. The best fit returns $\gamma=0.94_{-0.10}^{+0.09}$, suggesting a very hard source spectrum, and an injection of mostly intermediate mass nuclei, with very few protons or iron nuclei. It has to be noted that the fit also finds a second local minimum, with $\gamma \sim 2$ and a larger maximum rigidity, more in line with standard models of cosmic-ray acceleration. This solution is however disfavored since it predicts wider distributions of UHECR masses than observed in the data. Data relative to the energy spectrum alone cannot help us to identify the nature of cosmic rays. We showed that mass measurements can provide valuable information. Auger data indicate that, in addition to the propagation effect, the flux suppression may reveal the limiting energy of the most powerful cosmic particle accelerators but this would require hard injection spectra (unexpected) or close low luminosity sources. 

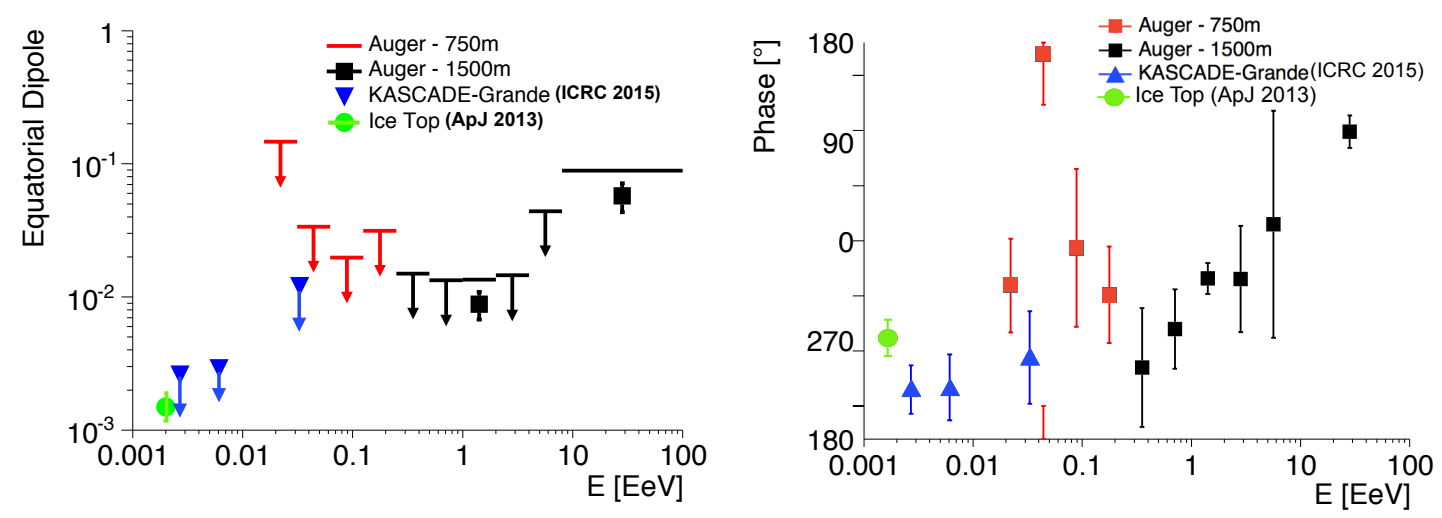

Figure 6: Measurements of the equatorial component of the dipole by Auger [21], KASCADE-Grande [23] and IceTop [24]. See [21] for further details.

\section{Searches for anisotropies}

Anisotropies appearing on large angular scale can be caused by the propagation of cosmic rays and/or by their source distributions in the sky. The search for dipolar or multipolar structures in the distribution of the observed arrival directions of cosmic rays is then complementary to spectrum and mass measurements. The Pierre Auger Collaboration has implemented different analyses to search for dipolar and quadrupolar anisotropies in different energy ranges spanning four orders of magnitude [21]. The used technique is based on the harmonic analysis of the counting rate. The current status of a prescribed test on the phase of the first harmonic modulation in right ascension is also discussed following a hint of a smooth phase transition from $270^{\circ}$ to $100^{\circ}$ above $4 \times 10^{18} \mathrm{eV}$ already published in [22]. A summary of the measurements of the equatorial amplitudes and of the phases in the wide energy range is shown in Fig. 6. When the dipole is not significative, an upper limit is reported. The measurements presented by the KASCADE-Grande collaboration [23] and Icetop [24] are also shown. The Auger analysis benefits from the inclusion of events at large zenith angles allowing a significative increase of the covered sky up to $85 \%$. At the highest energies, above $8 \times 10^{18} \mathrm{eV}$ the large-scale anisotropy has a significance exceeding $4 \sigma$.

Interestingly, the phase above $8 \mathrm{EeV}$, about $95^{\circ}$ in right ascension, is roughly opposite of the one at energies below $1 \mathrm{EeV}$, which is approximately directed towards of the Galactic Center. This fact may support the hyphotesis of an extra-galactic component gradually taking over a Galactic one even if this possibility has to be explored with additional data. The percent limits to the amplitude of the anisotropy exclude the presence of a large fraction of Galactic protons at $\mathrm{EeV}$ energies [25]. The large-scale distribution of arrival directions of cosmic rays has been also studied by combining the data of Auger and Telescope Array [26]. Thanks to the full-sky coverage, it has been possible to report the first angular power spectrum of cosmic rays above $10^{19} \mathrm{eV}$. The data show a significative dipole amplitude and no deviations from isotropy at smaller angular scales. The largest deviation from isotropy occurs for the dipolar moment. Its observed amplitude is $(6.5 \pm 1.9) \%$ with a chance probability of $5 \times 10^{-3}$, pointing to $\left(93^{\circ} \pm 24^{\circ}\right)$ in right ascension and $\left(-46^{\circ} \pm 18^{\circ}\right)$ in declination. This result is well in agreement with Auger-only data. Fig.7, left, shows the sky maps in equatorial coordinates of the flux for Auger and TA events with $\mathrm{E}>10 \mathrm{EeV}$. 

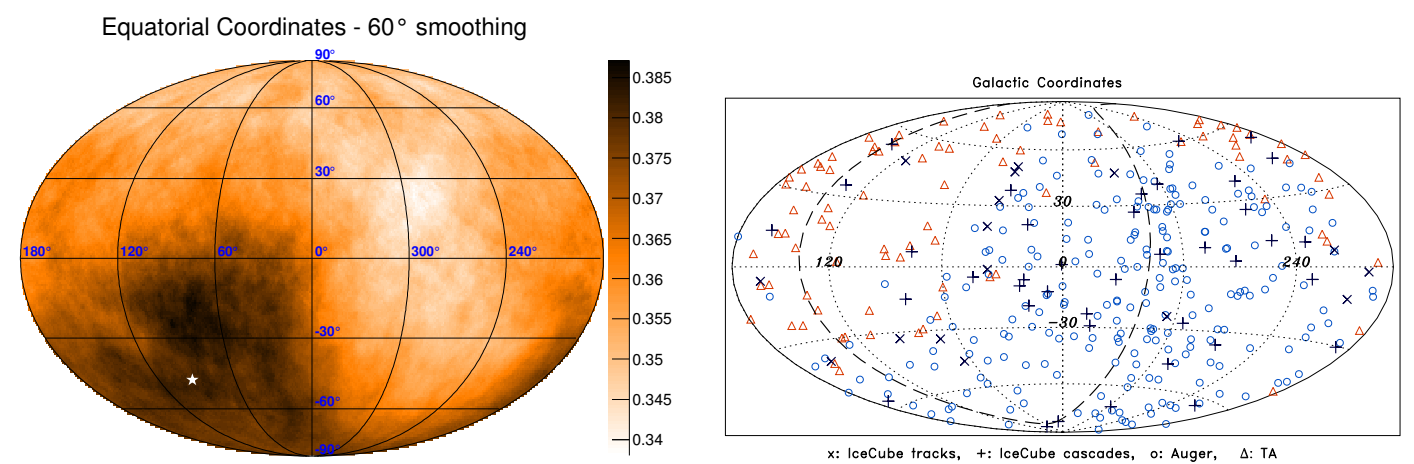

Figure 7: Left: Sky maps in equatorial coordinates of the flux, in $\mathrm{km}^{-2} \mathrm{yr}^{-1} \mathrm{sr}^{-1}$ units, smoothed in angular windows of $60^{\circ}$ radius, for Auger and TA events with E $>10 \mathrm{EeV}$. See [26] for details. Right:AitoffHammer projection of the sky in galactic coordinates showing the arrival directions of the IceCube highenergy cascades (plus signs) and high-energy tracks (crosses), and the UHECRs detected by Auger (circles) and TA (triangles). The dashed line indicates the super-galactic plane. See [27] for details.

The Auger collaboration has reported the results of a search for intermediate scale anisotropies above $4 \times 10^{19} \mathrm{eV}$ [27]. Cross-correlations with catalogs containing sources within $200 \mathrm{Mpc}$ give results compatible with isotropic expectations. The minima of the penalized probabilities in these searches are at the level of $1 \%$. One of the two largest deviations from isotropy (post-trial probability $1.4 \%$ ) is found (above $58 \mathrm{EeV}$ ) when looking within $15^{\circ}$ from the direction of Centaurus A, the closest radio-loud AGN. Other tests based on auto-correlation and on the search of excesses in circular windows along the sky yield negative results.

In the context of testing the distribution of the arrival directions of the highest-energy cosmic rays, it is worth mentioning the result of a full-sky study, originating from a fruitful collaboration among Auger, Telescope Array and IceCube [28]. It consists in the search for correlations between the directions of 318 UHECRs observed by the Auger Observatory ( 231 events with E $>52 \mathrm{EeV}$ ) and by the Telescope Array ( 87 events with $\mathrm{E}>57 \mathrm{EeV}$ ) and those of very high-energy neutrino candidates detected by IceCube (see Fig. 7 right, for a sky map of the events observed by the three experiments). Although no indications of correlations at discovery level are found, it is interesting to highlight that the smallest post-trial $p$-values (corresponding to $3 \sigma$ ) are obtained when considering the correlations between the directions of cascade events observed by IceCube and those of the UHECRs. The excess of correlations arises mostly from pairs of events in the region of the sky where the Telescope Array has reported an excess of events (so-called hot spot [29]) and in regions close to the super-galactic plane in correspondence with the largest excess observed in Auger data. The center of the hot spot is located at about $17^{\circ}$ from the super-galactic plane, in the vicinity of the Ursa Major cluster. Several potential candidates have been suggested as the source of the hot spot [30] but the current statistics are too low to draw any conclusion. Moreover the analysis is complicated by the big uncertainties on the deflections of cosmic rays during their propagation in the galactic and extra-galactic magnetic fields.

In summary, only small deviations from overall isotropic sky are observed. Either the deflections by magnetic fields are large, e.g. due to heavy primaries as supported by Auger composition studies, or the number of sources is very large. The lack of anisotropy at low energy suggest that 

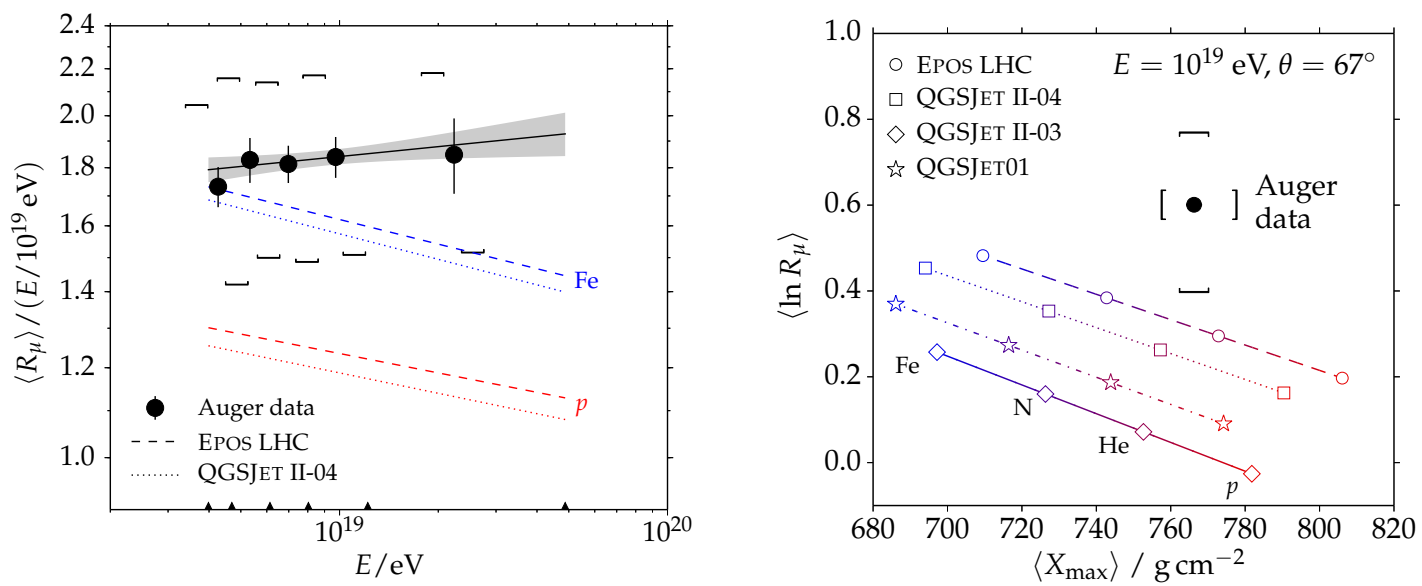

Figure 8: Measurements of the muon content of extensive air showers by the Auger collaboration [34].

protons (large fraction at ankle) are probably of extra-galactic origin (unless assuming extreme values of the galactic magnetic field). Currently all experiments do not have enough sensitivity at the highest energies to draw any conclusion on composition even with a better understanding of hadronic interactions.

\section{Hadronic physics}

We have shown in Sec. 4 that the measurement of mass composition is a crucial step to discriminate between different acceleration and propagation scenarios. Information about composition are currently derived using the fluorescence detection technique, through the measurement of $X_{\max }$ (see Sec. 3). However, the interpretation of data depend on the predictions of hadronic interaction models and conclusions are influenced by their uncertainties. Hadronic interaction models extrapolate at ultra-high energies all interaction properties measured by the accelerators in a lower energy domain (the $14 \mathrm{TeV}$ c.m.s. energy of the proton-proton collisions at LHC corresponds to $10^{17} \mathrm{eV}$ in the lab system). This makes the problem of the determination of mass composition very difficult to be addressed. At these energies, the hadronic multiparticle productions have to be parametrized by phenomenological models as they cannot be calculated from first principles using QCD theory, which is not perturbative at low transverse momenta. A review of the current status of the field can be found in [31]. Moreover, the fluorescence data suffer from small statistics due to the low measurement duty cycle $(\sim 15 \%)$. The hybrid design of the Pierre Auger Observatory and of the Telescope Array benefits of the combination of a fluorescence detector with a surface detector with a almost $100 \%$ duty cycle. This offers the possibility of using different methods based on observables that can be measured with the surface detector. In particular muon measurements are sensitive to the details of the hadronic component of the air shower and provide a handle to study the mass composition independently of $X_{\max }$. In this direction, the Pierre Auger Collaboration has published the study of the muon content at the ground [32] and the study of the atmospheric depth at which the muon production rate reaches a maximum in air showers [33]. More recently, a measurement of the muon shower size has been presented by the Auger Collaboration [34]. All these analyses 


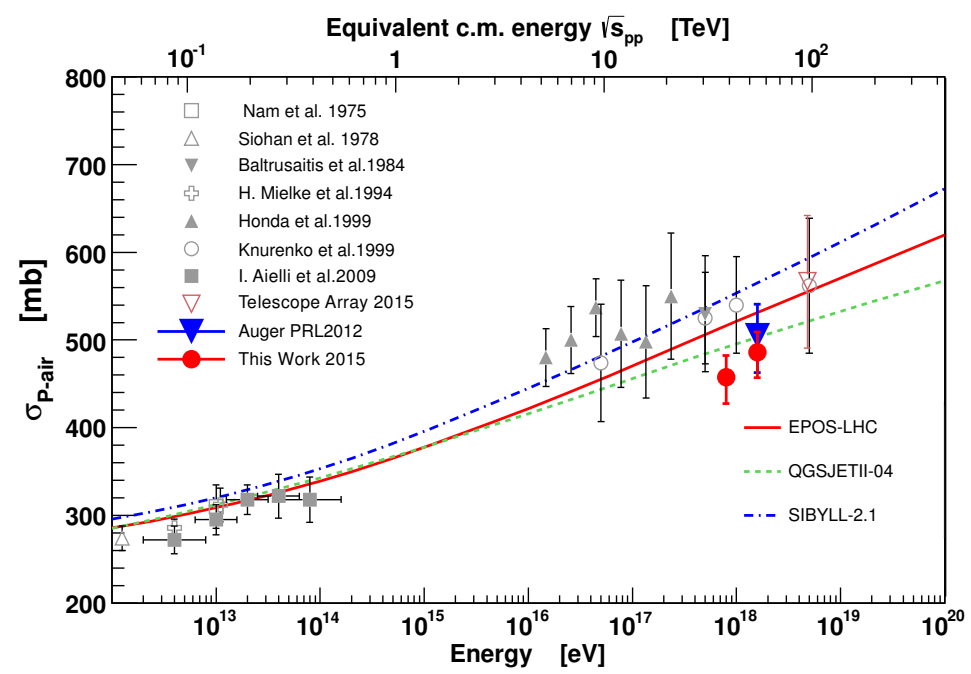

Figure 9: Measurement of the proton-air collision cross section from several experiments including the latest measurements from Auger [42] and TA [43].

rely on the fact that the electromagnetic component of inclined showers is largely absorbed in the atmosphere before reaching the ground. It has been shown that the shape of the distribution of the number of muons per unit area at the ground is almost independent of energy, composition or hadronic interaction model [35, 36]. In Fig. 8, left panel, the measured average muon content per shower as a function of primary energy is compared with the results of simulations using different hadronic interaction models. Models can hardly reproduce data, independently of composition. The space for agreement is really limited. The muon size in the data is even larger than the one predicted for iron when, from the $X_{\max }$ measurements, one would expect an average logarithmic mass between proton and iron, see Sec. 3. This tension is more evident in the measurement of the average logarithmic muon content as a function of the average $X_{\max }$ at $10^{19} \mathrm{eV}$ shown in the right panel of Fig. 8. Looking at the correlation between the muon size and $X_{\max }$ predicted by the various interaction models, one can realize how big the impact of LHC measurements (EPOS-LHC and QGSJetII-04 are the only models tuned with the LHC data) is for cosmic-ray physics.

Auger and TA have presented their new measurements of the proton-air collision cross section using the data of the fluorescence telescopes [42, 43]. The two collaborations use different analysis methods based on a common bottom line: the attenuation length of proton air showers derived from the $X_{\max }$ distribution is an observable that is directly correlated with the cross-section of inelastic proton-air collisions. The measured cross-sections are shown in Fig. 9. In order to reduce possible biases arising from a contamination of nuclei heavier than protons, the Auger measurements are limited to energies below $10^{18.5} \mathrm{eV}$. The current status and the perspective of the experiments measuring hadronic interactions at the LHC have been the subject of many presentations at this Conference. This demonstrates the good and productive connection to the community of particle and nuclear Physics. Relevant for the field of UHECR are for example the measurements of the proton-proton collision cross-sections and of the charged particle pseudorapidity distributions by TOTEM [37], the measurements of proton-lead cross-section from CMS [38] and of the production of neutral particles ( $\gamma, \pi^{0}$ and neutrons) in the very forward region by LHCf [39]. A new important 

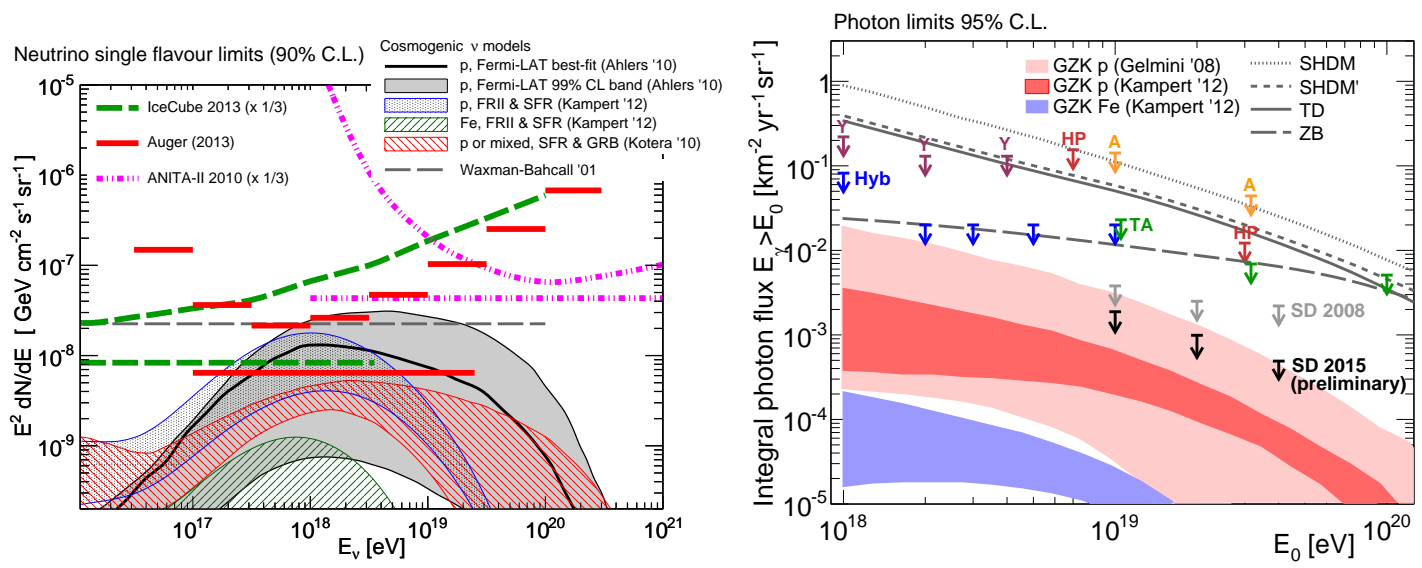

Figure 10: Left: Upper limits to the diffuse flux of UHE neutrinos at $90 \%$ C.L. in integrated (horizontal lines) and differential form compared to expectations. Right: Upper limits at 95\% C.L. to the diffuse flux of UHE photons and predictions from several top-down and cosmogenic photon models. See [47] for details and references to model prediction and experimental results.

measurement of the multiplicity of the $\rho^{0}$ meson in pion-carbon interactions has been made by the NA61/SHINE collaboration [40]. They have found that it is underestimated by all hadronic interaction models. The $\rho^{0}$ production plays a crucial role in the shower development since this meson decays to $\pi^{+} \pi^{-}$. By changing the relative weight of the $\rho^{0}$ and $\pi^{0}$ multiplicities one can change the relative weight of the muon and electromagnetic component of the shower. This is now implemented in a new preliminary version of the event generator Sybill [41].

\section{Multi-messengers from the Universe: Photons and Neutrinos}

The search for ultra-high energy primary photons and neutrinos is motivated by several arguments. From an astrophysical point of view, the evidence of their existence would open a new window on the most extreme Universe. The detection of neutrinos and photons, as eventually produced by the decay of charged and neutral pions respectively, would also provide an independent proof of the GZK-effect [44]. Independently of a positive or negative result, their search will help constraining astrophysical scenarios for the origin and the propagation of UHECR, exotic models (i.e., Super Heavy Dark Matter (SHDM), topological defects (TD), Z-burst, etc., see [45] for a review) and provide hints of fundamental and new physics (e.g. Lorentz Invariance Violation [46]). The Auger Collaboration has presented updated results on the search for photons and neutrinos as primary particles [47]. The search for neutrinos exploits the extremely small cross-section of the signal particles. At large zenith angles $\left(\theta>60^{\circ}\right)$ the thickness of the atmosphere traversed is large enough to absorb almost completely the electromagnetic component of showers initiated by nucleons or even photons, leaving their signal dominated by muons. Showers initiated by neutrinos very deep in the atmosphere, on the other hand, have a considerable amount of the electromagnetic component remaining ("young" showers). Downward-going neutrinos of all flavors are searched for at zenith angles $\theta>60^{\circ}$ and upward-going tau neutrinos at $\theta>90^{\circ}$. 
Showers induced by photons are characterised by a lower content of muons and larger average depth of maximum longitudinal development $X_{\max }$ than showers initiated by nuclei with the same energy. This is due to the radiation length being more than two orders of magnitude smaller than the mean free path for photo-nuclear interaction, causing a reduced transfer of energy to the hadron/muon channel, and to the development of the shower being delayed by the typically small multiplicity of electromagnetic interactions. Photons are searched for in data with zenith range $30^{\circ}$ $-60^{\circ}$ : the lower cut ensures that photon showers at these energies are fully developed at the depth of the SD. The selection criteria are additionally based on the lateral distribution of particles, steeper than in nuclear showers, due to the reduced muon content in photon showers. In both analyses the cosmic-ray background is not simulated, but determined through a fraction of data used as a training sample. After application of the selection criteria to data, no neutrino event survives the selection, while 4 events survive the photon search, compatible with background expectations.

The derived upper limits on their fluxes, assuming a differential flux $d N(E)=k E^{-2}$, are shown in Fig. 10, left and right for neutrinos and photons, respectively. Concerning Auger results, both neutrino and photon limits reach the values as predicted in case of a pure proton composition at the sources. Neutrino limits are below the Waxman-Bahcall bound [48], for the first time with data from an EAS array. The photon limits are also compared to the fluxes expected for the case of top-down models of UHECR production, e.g., from the decay of super-heavy relic particles from the early Universe: they appear to be clearly disfavored, suggesting source mechanisms for the acceleration of UHECRs.

\section{Upgrade}

Upgrade plans are planned for both the experiments, Auger and TA. The surface array of TA will be extended to cover an area of about $3000 \mathrm{~km}^{2}$. That will provide an acceptance close to the one of Auger in the Southern Hemisphere and it will allow the observation of the full sky with similar significance along the next years.

The Auger upgrade, named AugerPrime, aims at complementing the water-Cherenkov detectors of the surface array with scintillator detectors [49]. This will allow the determination of the muonic shower component, thus extending the composition sensitivity of the Auger Observatory into the flux suppression region.

Obtaining additional composition-sensitive information will not only help to better reconstruct the properties of the primary particles at the highest energies, but also improve on the measurements in the crucial energy range just above the ankle. Furthermore, measurements with the new detectors will help reducing systematic uncertainties related to hadronic models and reconstruction algorithms. Finally it should be mentioned that the addition of scintillator detectors across the entire Observatory will also make possible direct comparisons of Auger measurements with those of the surface detectors of the Telescope Array experiment. This will strengthen the already productive cooperation between the two collaborations, which has the aim of understanding the highest energy cosmic-ray flux across the entire sky. The design of the surface scintillator detectors (SSD) is simple, reliable and they can be easily deployed over the full $3000 \mathrm{~km}^{2}$ area of the overall Auger Surface Detector (SD). An SSD unit will consist of a box of $3.8 \mathrm{~m} \times 1.3 \mathrm{~m}$, housing two scintillator modules, each covering an area of $1.9 \mathrm{~m}^{2}$, see Fig. 11 (left). 

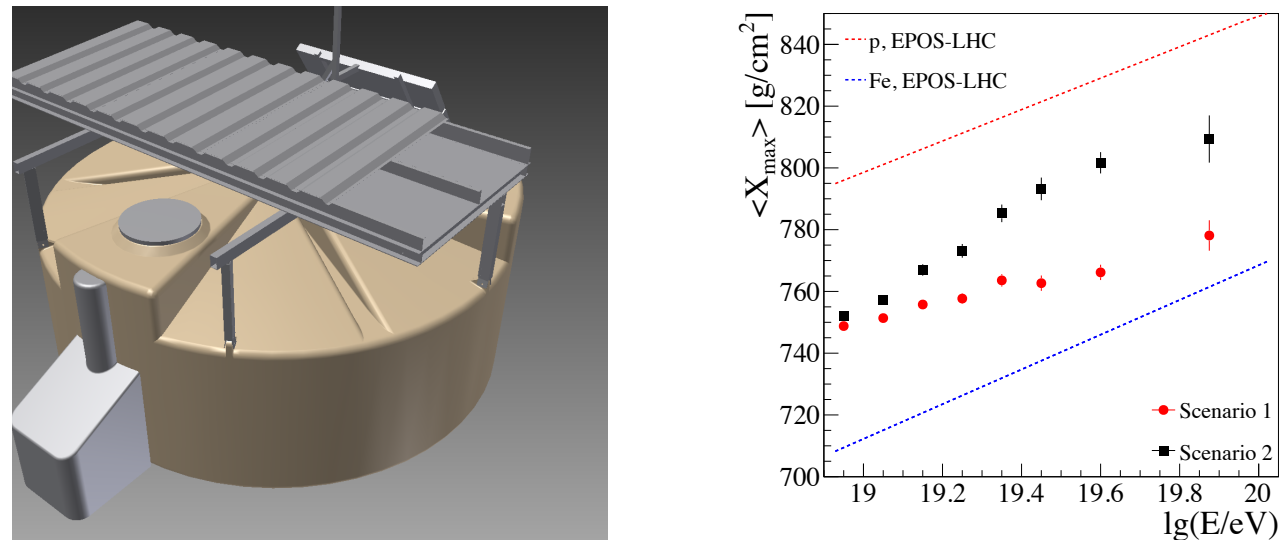

Figure 11: Left panel: 3D view of a SSD mounted on a WCD. A double roof, with the upper layer being corrugated aluminum (here shown partially cut away for clarity), is used to reduce the temperature variations. Right panel: Reconstructed mean depth of shower maximum $X_{\max }$ (left) using only SD data. Shown are the two scenarios: (1) maximum-rigidity model; (2) photo-disintegration model, see the text for details.

To demonstrate the potential of the upgraded detector we have chosen two physics motivated benchmark models [18] fitted to the Auger flux and composition data for $E>10^{18.7} \mathrm{eV}$. In Fig. 11 right, we show the expected discrimination power of the additional information. Mock data sets were generated for these scenarios with a statistics corresponding to 7 years of data taking with AugerPrime. Only SD data are used in the reconstruction.

The Auger upgrade, with operation planned from 2018 until 2024, will allow us to address some of the most pressing questions in UHECR physics, including that of the origin of the flux suppression, the prospects of light particle astronomy and secondary particle fluxes, and the possibility of new particle physics at extreme energies.

\section{References}

[1] The Pierre Auger Coll., Nucl. Instrum. Meth. A 798, 172 (2015)

[2] T. Abu-Zayyad et al. Telescope Array Coll., Nucl. Instrum. Meth. A 689, 87 (2012)

[3] P.L. Ghia for the Pierre Auger Coll., Highlight Talk, Proc. of the $34^{\text {th }}$ ICRC, The Hague (2015)

[4] C. Jui for the Telescope Array Coll., Highlight Talk, Proc. of the $34^{\text {th }}$ ICRC, The Hague (2015)

[5] I. Valiño, for the Pierre Auger Coll., Proc. of the $34^{\text {th }}$ ICRC, The Hague, PoS(ICRC2015)271

[6] V. Verzi, for the Pierre Auger Coll., Proc. of the $33^{\text {rd }}$ ICRC, Rio de Janeiro (2013)

[7] The Pierre Auger Coll., Astropart. Phys. 34, 368 (2011)

[8] D. Ivanov, for the Telescope Array Coll., Proc. of the $34^{\text {th }}$ ICRC, The Hague, PoS(ICRC2015)349

[9] V. Verzi, Rapporteur Talk, Proc. of the $34^{\text {th }}$ ICRC, The Hague, PoS(ICRC2015)349

[10] I. Mariş et al., for the Pierre Auger and TA Collbs., Proc. of UHECR2014, Springdale (2014)

[11] A. Porcelli, for the Pierre Auger Coll., Proc. of the $34^{\text {th }}$ ICRC, The Hague, PoS(ICRC2015)420

[12] The Pierre Auger Coll., Phys. Rev. D 90, 122005 (2014) 
[13] The Pierre Auger Coll., Phys. Rev. D 90, 122006 (2014)

[14] The Pierre Auger Coll., JCAP 1302, 026 (2013)

[15] M.Unger, for the Pierre Auger Coll., Proc. of the $34^{\text {th }}$ ICRC, The Hague, PoS(ICRC2015)307

[16] R.U. Abbasi et al. Telescope Array Coll., Astropart. Phys. 64, 49 (2014).

[17] E. Barcikowski et al. for the Pierre Auger, TA and Yakutsk Coll., EPJ Web Conf. 53, 01006 (2013)

[18] A. di Matteo, for the Pierre Auger Coll., Proc. of the $34^{\text {th }}$ ICRC, The Hague, PoS(ICRC2015)249

[19] R. Aloisio et al., JCAP 1210, 007 (2012)

[20] A. Schulz for the Pierre Auger Collababoration, Proc. of the $33^{\text {rd }}$ ICRC, Rio de Janeiro (2013)

[21] I. Al Samarai, for the Pierre Auger Coll., Proc. of the $34^{\text {th }}$ ICRC, The Hague, PoS(ICRC2015)372

[22] I. Sidelnik, for the Pierre Auger Coll., Proc. of the $33^{\text {rd }}$ ICRC, 582, Rio de Janeiro (2013)

[23] A. Chiavassa, for the KASCADE-Grande Coll., Proc. $34^{\text {th }}$ ICRC, The Hague, PoS(ICRC2015)281

[24] IceCube Coll., Astrophys. J. 765 55, (2013)

[25] The Pierre Auger Coll., Astrophys. J. Suppl. 203 (2012) 34

[26] O. Deligny, for the Pierre Auger and Telescope Array Collbs., PoS(ICRC2015)395

[27] J. Aublin, for the Pierre Auger Coll., Proc. of the $34^{\text {th }}$ ICRC, The Hague, PoS(ICRC2015)310

[28] G. Golup, for the Auger, TA and IceCube Collbs., Proc. $34^{\text {th }}$ ICRC, The Hague, PoS(ICRC2015) 1082

[29] K. Kawata et al., for the TA Coll., Proc. of the $34^{\text {th }}$ ICRC, The Hague, PoS(ICRC2015)276

[30] H. Haoning et al., Proc. of the $34^{\text {th }}$ ICRC, The Hague, PoS(ICRC2015)325

[31] R. Engel, Proc. of the $34^{\text {th }}$ ICRC, The Hague, PoS(ICRC2015)007

[32] The Pierre Auger Coll., Phys. Rev. D 91, 032003 (2015)

[33] The Pierre Auger Coll., Phys. Rev. D 90, 012012 (2014)

[34] L. Collica, for the Pierre Auger Coll., Proc. of the $34^{\text {th }}$ ICRC, The Hague, PoS(ICRC2015)336

[35] M. Ave et al., Astropart. Phys. 14, 91 (2000)

[36] H.P. Dembinski et al., Astropart. Phys. 34, 128 (2010)

[37] F. S. Cafagna, for the TOTEM Coll., Proc. of the $34^{\text {th }}$ ICRC, The Hague, PoS(ICRC2015)312

[38] C. Baus et al., for the CMS Coll., Proc. of the $34^{\text {th }}$ ICRC, The Hague, PoS(ICRC2015)440

[39] Y. Itow, for the LHCf Coll., Proc. of the $34^{\text {th }}$ ICRC, The Hague, PoS(ICRC2015)259

[40] A. E. Hervé, for the NA61/SHINE Coll., Proc. of the $34^{\text {th }}$ ICRC, The Hague, PoS(ICRC2015)330

[41] R. Engel et al., Proc. of the $34^{\text {th }}$ ICRC, The Hague, PoS(ICRC2015)558

[42] R. Ulrich, for the Pierre Auger Coll., Proc. of the $34^{\text {th }}$ ICRC, The Hague, PoS(ICRC2015)401

[43] R. Abbasi, J. Belz, for the TA Coll., Proc. of the $34^{\text {th }}$ ICRC, The Hague, PoS(ICRC2015)402

[44] Zatsepin G. T. and V. A. Kuz'min Zh. Eksp. Teor. Fiz. Pis'ma Red. 4, 144 (1966)

[45] P. Bhattacharjee, G. Sigl, Phys. Rep. 327, 109 (2000)

[46] M. Galaverni and G. Sigl, Phys. Rev. Lett. 100, 021102 (2008)

[47] C. Bleve, for the Pierre Auger Coll., Proc. of the $34^{\text {th }}$ ICRC, The Hague, PoS(ICRC2015)1103

[48] J. Bahcall and E. Waxman, Phys. Rev. D 64, 023002 (2001)

[49] R. Engel, for the Pierre Auger Coll., Proc. of the $34^{\text {th }}$ ICRC, The Hague, PoS(ICRC2015)686 